soluble MWFs, synthetic MWFs and polycyclic aromatic hydrocarbons (PAHs) not included in MWFs. Three quantitative time-varying metrics were used in the models: the duration, the frequency-weighted duration and the cumulative exposure index. Cox models were fitted with the standard partial likelihood approach using only the 3 countermatched controls for each case as well as by maximizing the pseudolikelihood which uses all the controls sampled for each case with calibrated weights.

Results Compared to the standard method, in the pseudolikelihood approach there is a reduction in the variance of the estimates for straight MWFs, synthetic MWFs and smoking but an increase for soluble MWFs and PAH. The hazard ratios that were $>1$ with the standard method were attenuated when considering the pseudolikelihood approach. The hazard ratios for the straight MWFs in the pseudolikelihood approach were 1.10 (95\% CI: 1.01 - 1.19) per year of exposure and 1.33 (95\% CI: 1.05 - 1.7) per full-time equivalent year of exposure.

Conclusion Nested case-control study under countermatching design would benefit from pseudolikelihood approach. Results from the current study suggest that occupational exposure to straight MWFs increases the risk of bladder cancer.

\section{0-300 CORRECTING THE REFERENCE LIFE TABLE IN MORTALITY ANALYSIS: APPLICATION IN A COHORT OF SEWER WORKERS}

${ }^{1}$ Michel Grzebyk, Isabelle Clerc-Urmès, Eve Bourgkard, Guy Hédelin. ${ }^{1}$ French National Research and Safety Institute, France

\subsection{6/OEM-2021-EPI.82}

Objectives To apply correction in life tables in mortality analyses to address selection effect with respect to the reference population in a cohort of sewer workers.

Methods We used excess hazard model to assess the excess mortality from all-cause and from all-cancer in an historical cohort of 1898 sewer workers between 1960 and 2011. National and regional life tables were available to assess the all-cause and the all-cancer background mortality. The corrections of these life tables were modeled by spline functions in the logit of survival scale. The excess hazard was modeled using splines functions with the time since hiring as the time scale. The parameters of the model were estimated by maximizing the likelihood. The expected excess number of cases were estimated using both regional and national model-based corrected tables and compared to those obtained without correcting the life tables. In a simulation study estimates were obtained using the corrected life table with a known model of correction (i), using the uncorrected life table but applying the model of correction (ii), and without model of correction (iii). Results The simulation study showed that applying the model of correction reduces the estimation bias in the excess rate model. In the cohort study, for all-cause mortality, the difference between the excess numbers of cases estimated reduced from 28.0 using the original life tables to 1.5 when a model of correction was applied. For all-cancer mortality, the difference reduced from 24.3 to 11.8 . However, the standard error was doubled.

Conclusions The differences between estimates obtained using two reference life tables decreased when the model of correction was applied at a cost of larger confidence intervals.
Correction in life tables can be applied in mortality analyses when the life tables available are not fully suitable to the cohort studied.

\section{0-304 CONCORDANCE BETWEEN THE CANADIAN JOB- EXPOSURE MATRIX (CANJEM) AND EXPERT ASSESSMENT IN OCCUPATIONAL EXPOSURE ASSESSMENT AMONG JOBS HELD BY WOMEN}

${ }^{1}$ Mengting Xu, Vikki Ho, Jérôme Lavoué, Lesley Richardson, Jack Siemiatycki. 'University of Montreal, Canada

\subsection{6/OEM-2021-EPI.83}

Introduction The Canadian job-exposure matrix (CANJEM) is a general population JEM built from expert assessment data of 31,673 jobs held by 8,760 participants from four Montreal case-control studies.

Objective To examine the validity of CANJEM for jobs held by women, by comparing exposure assessments using CANJEM and our expert assessment method to a selected list of 69 agents.

Methods We compared the exposure estimates for 69 agents within a population-based case-control study of lung cancer assigned by expert assessment to those derived from CANJEM. We linked the job histories of 998 women (3403 jobs) to CANJEM and thereby, derived probability of exposure to each of the 69 selected agents in each job. To create binary exposure variables (exposed/unexposed), we dichotomised probability of exposure using two cutpoints: $25 \%$ and $50 \%$ (referred to as CANJEM-25\% and CANJEM-50\%). Using the 3403 jobs as units of observation, we estimated the prevalence of exposure to each selected agent using CANJEM-25\% and CANJEM-50\%, and using expert assessment. Further, using expert assessment as the gold standard, for each agent, we estimated sensitivity, specificity and Kappa.

Results CANJEM-based prevalence estimates correlated well with the prevalences assessed by the experts. Sensitivity, specificity and Kappa varied greatly among agents, and between CANJEM-25\% and CANJEM-50\% probability of exposure. For some agents such as fabric dust and cooking fumes, the concordance between CANJEM-based and expert-based assessments was high and inspired confidence that CANJEM-based assessments will be adequate; however for many other agents, the concordance was low. We present concordance estimates for 69 agents.

Conclusion Exposure concordance measures between CANJEM and expert assessment differed greatly by agents. The results of this study could guide users of CANJEM as to which agents are most likely to provide results that mimic those that would be obtained with expert assessment.

\section{0-467 EVALUATING THE IMPACT OF SEX AND GENDER ON THE PERFORMANCE OF MACHINE LEARNING FOR AUTO ENCODING OF JOB TITLES}

Christopher Baker, Anil Adisesh, Cesar Augusto Suarez, Ellen Sweeney, 'Amanda Von Seehausen, Mohammad Sadnan Al Manir, Deobrah Addey, Yunsong Cui, Hicks Jason, Cheryl Peters. 'University of New Brunswick, Canada

\subsection{6/OEM-2021-EPI.84}

Introduction Ongoing studies into the use of algorithms for the automated coding of job titles to the Canadian National 
Occupation Classification have performance accuracy which are at least equivalent to manual coding accuracy. Moreover automated coding provides significant time savings. These studies have identified that both natural language processing and machine learning algorithms are effective for auto coding. Whereas NLP based and machine learning approaches both rely on bespoke rules, and existing data sets, machine learning models can proliferate bias from training data if not corrected.

Objectives The goal of the study is to explore the impact of altering sex/gender ratios in training data sets on overall performance of the machine learning based prediction of NOC codes using patient provided job titles.

Methods Using data participant patient data provided by Atlantic PATH, training data sets were prepared for 100 4digit NOC categories. The data sets were prepared with sex/ gender ratios of 50/50 30/70, 70/30. The data sets were used to train ENENOC machine learning platform and tested on a set of manually coded job titles provided by Atlantic PATH CanPATH . Performance levels were contrasted for all 4-digit NOC categories used in the study.

Results Initial results in this preliminary study have identified that sex and gender are variables that can influence auto coding performance, however the extent to which overall coding accuracy is impacted is relative minor. Further studies are required with larger training sets to fully explore the extent of sex and gender as contributing variables to bias to ENENOC.

Conclusion We initiated studies to investigate the impact of sex and gender bias on performance of the ENENOC algorithm. Together, the ENENOC contributed training and test sets provide a suitable framework for ongoing work in this area.

\section{Muskuloskeletal-1}

\section{0-21 PATTERNS OF OPIOID DISPENSING AND ASSOCIATED WAGE REPLACEMENT DURATION IN WORKERS WITH ACCEPTED CLAIMS FOR LOW BACK PAIN: A RETROSPECTIVE COHORT STUDY}

${ }^{1}$ Michael Di Donato, Ting Xia, Ross lles, Rachelle Buchbinder, Alex Collie. 'Monash University, Australia

\subsection{6/OEM-2021-EPI.85}

Introduction When unable to work due to low back pain a worker may seek income support and funding for healthcare from an income support system such as workers' compensation. Clinical practice guidelines recommend opioids for low back pain are only used for a short duration, at a low dose and with a plan to cease use.

Objectives This study aimed to identify patterns of opioid dispensing in Australian workers with low back pain and determine the association of dispensing patterns with wage replacement duration.

Methods Australian workers' compensation claimants with low back pain and at least one day of wage replacement were included. We used group-based trajectory modelling to identify opioid dispensing patterns over a two and half year period from reported low back pain onset. Quantile regression was then used to compare wage replacement duration between each dispensing pattern group.
Results One third of workers with low back pain $(\mathrm{N}=3205$, $33.3 \%$ ) received at least one opioid dispense during their claim. Three dispensing patterns were identified. The majority had a short-term low-volume opioid dispensing pattern $(\mathrm{N}=2166,67.6 \%)$, while $798(24.9 \%)$ had a long-term moderate-volume pattern and $241(7.5 \%)$ had a long-term highvolume pattern. Workers dispensed opioids had significantly longer wage replacement duration than those not dispensed opioids (median (weeks): 63.6 versus 7.1 respectively). In addition, moderate- and high-volume long-term dispensing had significantly longer wage replacement duration compared with short-term dispensing (median (weeks): 126.9, 126.0 and 30.7 respectively).

Conclusion Our study found a high use of opioids for long durations among compensated Australian workers with low back pain. Multifaceted strategies to limit long-term use of opioids are needed. These could include implementation of clinical care standards and indicators that can be used to monitor and regulate opioid use, and implementing financial mechanisms to stem long-term opioid use.

\section{0-179 OCCUPATIONAL DEMANDS ASSOCIATED WITH ROTATOR CUFF DISEASE SURGERY: RESULTS FROM A NOVEL LINKAGE OF A JOB-EXPOSURE MATRIX TO THE UK BIOBANK}

${ }^{1}$ Elizabeth Yanik, Bradley Evanoff, Karen Walker-Bone, Ann Marie Dale, Yinjiao Ma, Jay Keener, Nitin Jain, Graham Colditz, Martin Stevens, Nancy Saccone, Rick Wright. ${ }^{1}$ Washington University in St. Louis, United States

\subsection{6/OEM-2021-EPI.86}

Objective Occupations requiring high physical demands may lead to greater risk of rotator cuff disease (RCD) and corresponding surgical treatment. We linked a job-exposure matrix (JEM) to the UK Biobank to measure physical occupational exposures and estimate associations with incident RCD surgery.

Methods Job titles at baseline and UK Standard Occupational Classification (SOC) codes were recorded during a verbal interview. Lifetime job histories were captured through a webbased survey. UK SOC codes were linked to a JEM based on the US O*NET database. O*NET-based scores for physical demands were assigned to jobs including: static strength, dynamic strength, general physical activities, handling/moving objects (range $=1-7$ ), time spent using hands, whole body vibration, and cramped/awkward positions (range=1-5). RCD surgeries were identified through national hospital inpatient records. Cox regression was used to calculate hazard ratios (HRs) as estimates of associations with RCD surgery accounting for confounders. Among those with lifetime job histories, associations were estimated with duration of time with high exposure (i.e. above cut-offs identifying approximately the top quartile of exposure).

Results Job titles were available for 277,808 people, of which $1,345(0.5 \%)$ had a subsequent inpatient RCD surgery. After adjusting for age, sex, race, education, deprivation, and body mass index, all O*NET variables considered were associated with RCD surgery (HR per point increase range $=1.10-1.45$, all $\mathrm{P}<0.005)$. More frequent occupational manual labor selfreported in the UK Biobank verbal interview was also associated with RCD surgery (HR for 'Always' vs. 'Never/ rarely' $=2.12 ; 95 \% \mathrm{CI}=1.79-2.50)$. Lifetime job histories were available for 100,929 people, in which high exposures were 